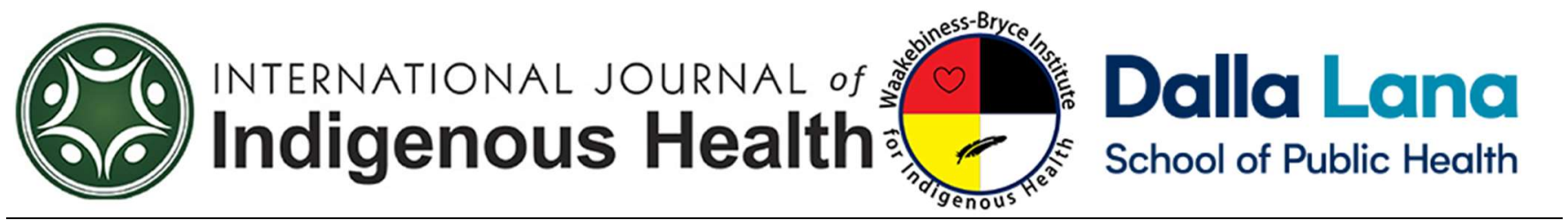

Volume 14

Issue 1. Physical Activity and Cultural Safety

Article 1

DOI 10.32799/ijih.v14i1.31944

May 2019

\title{
Community-Specific Risk and Protective Factors for Risky Alcohol Consumption in American Indian Women of Reproductive Potential: Informing Interventions
}

Annika C Montag, PhD.

Department of Pediatrics, University of California San Diego 9500 Gilman Drive MC 0828, La Jolla, CA 92093-

0828. Phone: 858-246-1755, email: amontag@ucsd.edu

Dan J Calac, MD.

Southern California Tribal Health Clinic, California

Christina D Chambers, PhD, MPH.

Departments of Pediatrics and Family Medicine and Public Health, University of California San Diego.

\section{Acknowledgements}

This research was supported by the National Institute of General Medical Sciences (GM087518) and the Indian Health Service (U26IHS300292). The authors are grateful to the American Indian Alaska Native women, Southern California Tribal Health Clinic staff, and project consultants for their valuable input and participation, and to our outstanding, insightful, knowledgeable, and hard-working research assistants.

Follow the International Journal of Indigenous Health at:

https://jps.library.utoronto.ca/index.php/ijih/index

\section{Recommended Citation}

Montag, A., Calac, D., Chambers, C. (2019). Community-Specific Risk and Protective Factors for Risky Alcohol Consumption in American Indian Women of Reproductive Potential: Informing Interventions. IJIH, 14(1), 8-28. DOI: $10.32799 /$ ijih.v14i1.31944 


\title{
Community-Specific Risk and Protective Factors for Risky Alcohol Consumption in American Indian Women of Reproductive Potential: Informing Interventions
}

\begin{abstract}
Objective: To explore the effect of community-specific risk and protective factors on risky alcohol consumption and vulnerability to having an alcohol-exposed pregnancy in women within a Southern California American Indian community. Methods: A sample of 343 American Indian women of childbearing age was enrolled in a study of risky drinking. All participants completed a questionnaire including alcohol consumption, other health behaviors, the T-ACE risky alcohol consumption screen and the PHQ-9 to measure depression and functionality. A subset of 80 women additionally answered focus group-derived questions about why they choose or do not choose to drink. Results: Risk and protective factors varied among sample subgroups. Broadly, factors affecting risk and protection included: depression, perception of other women's drinking, children/family, perception of risk to the unborn child, and feeling pressured to drink. Women's drinking was highly influenced by female friends and relatives. Women were most likely to drink with a girlfriend. Nearly $40 \%$ of all participants asked felt pressured to drink. Depression was associated with riskier alcohol consumption, less effective contraception, and testing positive for risky drinking using the T-ACE screen. Depressed women were more likely to binge drink because of stress, trauma, and "to escape my problems", and more likely to have been exposed to trauma including sexual assault. Conclusions: Interventions should incorporate community-specific factors. In the present sample, two separate strategies are indicated by the data: an information campaign to increase women's awareness of true social norms and the risks of prenatal alcohol-exposure; and screening for and treating depression.
\end{abstract}

\section{Keywords}

Alcohol, women, AIAN, prevention research, fetal alcohol spectrum disorders

\section{Creative Commons Licence

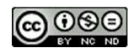

This work is licensed under a Creative Commons Attribution-Noncommercial-No Derivative Works 4.0 License. 


\section{Introduction}

Alcohol exposed pregnancies result in a range of persistent cognitive, behavioral and adaptive function deficits termed Fetal Alcohol Spectrum Disorders (FASD) (Jones \& Smith, 1973; Riley et al., 2011; Secretary of Health and Human Services, 2000; Streissguth, 2007). Interventions to prevent risky alcohol consumption and FASD may be more effective when specifically targeted (Caldwell, 2005; Fisher et al., 2007; Griner \& Smith, 2006; Kreuter et al., 2000; Montag et al., 2012; Skinner et al., 1999). For this reason, a good understanding of both risk and protective factors for risky alcohol consumption among women of reproductive potential is of high importance.

In the general population, the strongest known risk factor for an alcohol-exposed pregnancy (AEP) is drinking prior to pregnancy (Ethen et al., 2009; Palma et al., 2007). Other risk factors often cited but not always significantly associated include higher maternal age, gravidity and parity; being unmarried or unemployed; heavy alcohol consumption by spouse or partner; psychological stress; being less religious or spiritual; smoking; and illegal drug use (Bakhireva et al., 2011; Cannon et al., 2015; Flynn \& Chermack, 2008; Green PP, 2016; McLeod, 1993; Naimi et al., 2003; Palma, et al., 2007; Tsai et al., 2009; Waterson et al., 1990). Depression has also been associated with problem alcohol consumption in women (Helzer \& Pryzbeck, 1988; Kessler et al., 1997; KUO et al., 2006; Parker et al., 2010; Slade et al., 2013; Tsai, et al., 2009).

In addition, cultural norms influence the consumption of alcohol leading to varying vulnerabilities to an AEP (Balachova et al., 2012; Green PP, 2016; Nilsen et al., 2008; O'Keeffe et al., 2015; Roozen et al., 2016). Variations in these norms are of high relevance in the various distinct American Indian/Alaskan Native (AIAN) populations across the U.S. (Beals et al., 2003; May \& Gossage, 2001; O'Connell et al., 2005; Spicer et al., 2003; Yuan et al., 2010). However, risk factors for an AEP have not been well characterized for these communities. Similar to the general population, depression in AIAN communities may play a role (Beals et al., 2005; Dillard et al., 2012; Duran et al., 2004; Gone \& Trimble, 2012); and among several AIAN population samples, problem drinking and depression have been linked (Dillard, et al., 2012; Kunitz, 2006; Montag et al., 2015; O'Connell et al., 2006). As in other communities, healthcare provider advice may be largely ignored (O'Connor \& Whaley, 2006a) and women may not understand the extent to which they are at risk (Kaskutas, 2000), nor appreciate the true amount of alcohol they consume (Branco \& Kaskutas, 2001).

In addition to risk factors shared by the general population, there may be other risk and resiliency factors specific to AIAN communities (Allen et al., 2006; Hawkins et al., 2004; Whitbeck et al., 2004). Historical loss, trauma, and discrimination resonate throughout AIAN life today, and mistrust of research and externally imposed interventions may be important factors (Caldwell, 2005; Davis, 2002; Szlemko et al., 2006; Whitbeck, et al., 2004). Conversely, enculturation may be a source of resilience (Gray et al., 2010; Torres Stone et al., 2006; Whitbeck, et al., 2004), and family and community may exert protective influences for an AEP (LaFromboise et al., 2006). 
In this manuscript we describe the risk and protective factors in a specific AIAN community for risky drinking and an AEP using data from a study of AIAN women of reproductive age. This community-based study can inform prevention strategies specifically targeted to the risk and protective factors for that community. Given the known variability in AIAN communities' characteristics, an intervention's potential to succeed may depend upon the degree to which it is grounded in communityspecific factors.

\section{Methods}

\section{Ethics}

This study was approved by University of California at San Diego (UCSD), San Diego State University (SDSU), and Southern California Tribal Health Clinic (SCTHC) Institutional Review Boards. A Certificate of Confidentiality $(\mathrm{CoC})$, obtained from the National Institutes of Health (NIH), further protected the confidentiality of participants' data. All participants provided informed consent through a process where the consent form was read aloud and all staff members completed human subjects' protections training.

\section{Sample Source}

American Indian Alaska Native (AIAN) women from 18 to 45 years of age, of childbearing potential, were recruited from one of three AIAN health clinics located in Southern California between April 2011 and April 2014.

\section{Recruitment and Study Protocol}

Potential participants were approached by project staff, local AIAN community members, in waiting areas of health clinics and screened for eligibility. Interested and eligible participants were brought to a private room where they were taken through the consenting procedure, assigned a unique identifier, and completed a self-administered paper and pencil survey. The majority of women who completed the paper-based survey then completed a web-based survey, while a randomly selected subset was instead asked to complete an additional paper-based survey. Participants had the opportunity to be referred for professional substance problem use treatment following completion of each survey. Participants were provided incentives in the form of a $\$ 10$ gift card and a choice of a project fan or tshirt emblazoned with the project logo at baseline.

\section{Data Collection}

Data was collected by project staff members who were trusted local AIAN community members. 


\section{Entire sample}

Paper and pencil questionnaire. The self-administered, paper-based questionnaire included questions regarding current relationship situation (an eight response option question reduced to "cohabitating" or not), employment, religiosity, gravidity, parity, birth control use and frequency of correct usage, current use of prescription and non-prescription medications, smoking, illegal drug use, awareness of FASD, and alcohol consumption including number of standard drinks consumed per week and per occasion, and number of binge episodes in the past two weeks (binge defined in this setting as consuming $\geq 3$ standard drinks/occasion). They were asked to complete a T-ACE and a PHQ-9 questionnaire.

\section{Sample subgroups}

Web-based questionnaire. Participants selected for the web-based survey self-reported additional information by computer regarding alcohol consumption over the past two weeks, pregnancy status, family history of alcohol problems, the age they began drinking alcohol, and contraceptive use. Illustrations of various alcoholic beverage containers were used to prompt recall.

Paper and pencil questionnaire. These questions were the result of feedback from the community obtained through a focus group and interview process described in detail elsewhere (Montag et al., 2017). Topics included whether and, if yes, when women felt pressured to drink; why they drank when they binge drank and why they chose not to when they did not drink; who they drank with when they were binge drinking and questions about this person's drinking; and whether they had experienced trauma or abuse. At each point of contact, participants were offered referrals for professional treatment.

\section{Measures}

The nine item Patient Health Questionnaire (PHQ-9) measured depression and functionality. In a variety of studies it has been found to have a sensitivity of $73 \%$ and specificity of $94 \%$ for major depression (Kroenke \& Spitzer, 2002; Kroenke et al., 2001; Spitzer et al., 1999). In 2016, the PHQ-9 was adopted as a HEDIS (Healthcare Effectiveness Data and Information Set) measure of depression and incorporated into the majority of healthcare plans in the US. Despite extensive validation among other groups and use within AIAN communities, the PHQ-9 has not been specifically validated among AIAN women of childbearing age. However, using NHANES data, the PHQ-9 was found to be invariant across race/ethnicity and education level groups (Patel, 2017). Depression variables derived from this measure included "depression" (depressed or not depressed), and "functionality" (impaired or not impaired) and were calculated as follows: Depression: a participant scoring $>5$ was identified as "depressed"; Functionality: a participant scoring 2 or 3 was identified as having their "functionality impaired". 
T-ACE (Tolerance, Annoyed, Cut down, Eye-opener) is a validated screening instrument of four questions structured to identify risky drinking (Sokol et al., 1989) shown to be valid in an AIAN population (Gale et al., 1998). The T-ACE questions and scoring were as follows: How many drinks does it take to make you feel tipsy/high? ( $\leq 2=0$ points, $>2=2$ points); Have people annoyed you by criticizing your drinking? (No = 0 points, Yes = 1 point); Have you felt you ought to cut down on your drinking? (No $=0$ points, Yes $=1$ point); Have you ever had a drink first thing in the morning to steady your nerves or to get rid of a hangover? ( $\mathrm{No}=0$ points, $\mathrm{Yes}=1$ point). A score of $\geq 2$ was considered positive for risky drinking ("T-ACE positive" variable). Sensitivity of the instrument has been estimated at $80-90 \%$ and specificity at $40-70 \%$ (Chang, 2001; Chang et al., 1998; Chiodo et al., 2010; Russell et al., 1994; Russell et al., 1996).

Contraceptive effectiveness defined as by WHO (see http://www.fphandbook.org/). Using Birth Control Correctly was defined as responding that birth control was used correctly every time or most of the time, as opposed to some of the time or none of the time.

The "Vulnerability to Alcohol-Exposed Pregnancy" variable was defined in four categories: "not at risk", "at risk", "not at high risk", and "at high risk". Being "at risk" for an AEP was defined as 1) currently using alcohol and 2) using a less than highly effective contraceptive method. The NIAAA defines "risky drinking" for women as more than 3 drinks at one time or more than 7 drinks per week (NIAAA, 2011). For the purposes of this study, we defined "binge" or "risky" drinking as 3 or more standard drinks per occasion and/or 8 or more drinks per week as this level of consumption has been predictive of risk of adverse pregnancy outcomes in other studies (May et al., 2013; May et al., 2007; May et al., 2008; May et al., 2004; May \& Phillip Gossage, 2011). Being "at high risk" for an AEP was defined as "at risk" coupled with either consuming $\geq 3$ drinks per occasion or consuming $\geq 8$ drinks per week. The categories of vulnerability to AEP were not mutually exclusive, i.e., all "at high risk" women were also included in the "at risk" category.

"Regret" was measured by asking "Imagine you drank alcohol while you were pregnant and your child was born with fetal alcohol syndrome. How much would you regret drinking during your pregnancy?"

\section{Statistical Analyses}

Comparison of continuous, dichotomous, or categorical variables in tables of demographics, alcohol consumption, birth control use, knowledge questions (Appendix C), and depression were conducted using t-tests (continuous), $\chi 2$ (dichotomous), Fisher's exact test (dichotomous with small cell sizes), ANOVA, or nonparametric analyses (for data not normally distributed and not log-transformed). Normality in continuous variables was investigated by looking at skewness and kurtosis. ANOVA was used to examine associations among population characteristics. Regression was used to test for predictors of depression / impaired functionality and for vulnerability to AEP. First, each predictor was 
tested to determine whether there were significant independent associations with that factor and depression/ functionality/ vulnerability to AEP. Then multiple logistic regression analysis was used to examine all variables previously found to be significant. All two-way interactions among significant variables were tested. Statistical significance was defined as 2 -sided, p-value of $<0.05$. Statistical analyses were carried out using SPSS (PASW 18, SPSS Inc., Chicago, IL).

\section{Results}

\section{Total Sample}

A total of 343 women were recruited into the study, 33 of whom were pregnant. All women were included in analysis and tables indicate number responding to each question. Half of participants reported not currently consuming alcohol. As shown in Table 1 (see Appendix A), those reporting that they currently consumed alcohol were more likely to be employed, to have had more pregnancies, to use effective birth control and use it correctly, to smoke tobacco and to take illegal drugs. Drinkers and nondrinkers did not differ in the proportion who wanted more children, who were religious, or who were cohabitating. Furthermore, they did not differ in proportion identified as depressed or functionally impaired, or in FASD awareness.

Participants who did drink tended to drink in a heavy episodic pattern (binge drinking). Of 113 women responding to T-ACE questions, 73.5\% (a quarter of the total sample) were T-ACE positive, identified as a risky drinker. A quarter of participants used no form of contraception (including abstinence) and less than a quarter used highly effective birth control. Applying the definitions specified in the methods section, we found $42.3 \%$ of participants to be "at risk" of having an alcohol-exposed pregnancy and more than a third, 35.2\%, "at high risk" of AEP.

The level of awareness of FASD (nearly $3 / 4$ of participants had heard of FAS or FASD) and familiarity with community members affected by prenatal alcohol exposure (more than a third of participants knew someone affected) did not different across the study subgroups (Tables 1 and 2, see Appendix B).

The level of knowledge regarding the risks of alcohol to pregnancies was relatively high. Risky drinkers were slightly less likely to answer questions correctly (Table 3 ). The level of knowledge regarding the risks of alcohol to women and prevalence of local alcohol consumption was relatively low and not different across the study subgroups. 
Table 3.

Knowledge Questions (percent answering correctly)

\begin{tabular}{|c|c|c|c|c|c|}
\hline Type Questions & Comparator Group & $\mathbf{n}$ & Selected Subgroup & $\mathbf{n}$ & p-value \\
\hline & Not current drinker & & Current drinker & & \\
\hline Relating to Pregnancy & 95.9 & 62 & 92.0 & 58 & .040 \\
\hline Relating to Women's Health & 34.4 & 61 & 31.0 & 58 & .542 \\
\hline \multirow[t]{2}{*}{ Total } & 84.6 & 62 & 80.9 & 58 & .043 \\
\hline & Not risky drinker & & Risky drinker & & \\
\hline Relating to Pregnancy & 95.8 & 69 & 91.5 & 51 & .025 \\
\hline Relating to Women's Health & 36.0 & 68 & 28.4 & 51 & .175 \\
\hline \multirow[t]{2}{*}{ Total } & 84.8 & 69 & 80.0 & 51 & .009 \\
\hline & Not depressed & & Depressed & & \\
\hline Relating to Pregnancy & 94.7 & 78 & 92.8 & 40 & .339 \\
\hline Relating to Women's Health & 33.3 & 78 & 30.8 & 39 & .668 \\
\hline Total & 83.5 & 78 & 81.3 & 40 & .263 \\
\hline
\end{tabular}

More than a third of our sample (115 women) was identified as depressed. The proportion of participants categorized as depressed did not differ among current drinkers and abstainers. Nearly $14 \%$ of these women reported that their functionality was impaired. Women identified as depressed were more likely to consume alcohol in a risky fashion and to perceive a higher social norm regarding how many drinks are consumed by women of childbearing age in their community than women not identified as depressed (Table 2). In addition, they were less likely to use birth control (trend) and less likely to use it effectively; more likely to take prescription and illegal drugs; and more likely to test positive for risky drinking using the T-ACE screen. In this study, depression was associated with risk factors for having an alcohol-exposed pregnancy.

\section{Subset Sample}

Among the 80 women in our subset sample, 39\% reported feeling pressured to drink. The times women reported feeling most pressured to drink were "Girl's Night Out" (22.4\% of total and 56.7\% of those feeling pressured), "Holidays/occasions" (22.4\%), and "when I'm at the casino" (7.9\%).

When binge drinking, women were by far most likely to drink with a girlfriend, followed by partner/spouse, sister, and cousin (Table 4). This is true among the sample of all women responding to the question "On occasions when you drink 3 or more drinks in one sitting, who are you most often drinking with?" (n=69) and among the more limited sample of current drinkers responding $(n=51)$. The 
proportion choosing to binge drink with a girlfriend increased as the level of risk for an alcohol-exposed pregnancy increased. More risky drinkers and women at high risk for an AEP responded that they drank with a girlfriend than those not identified as risky drinkers or at high risk for AEP. While depressed participants reported binge drinking with a girlfriend more than with anyone else, they were statistically less likely to drink with a girlfriend than non-depressed participants $(\mathrm{p}=.038)$.

Table 4.

The Person Participants Reported Drinking with when Binge Drinking. (Percent among indicated subsample of 69 persons responding.)

\begin{tabular}{|lccccc|} 
Person drinking with & $\begin{array}{c}\text { Total Sample } \\
\mathbf{N}=\mathbf{6 9}\end{array}$ & $\begin{array}{c}\text { Current } \\
\text { drinkers }\end{array}$ & $\begin{array}{c}\text { Risky } \\
\text { drinkers } \\
\mathbf{n = 3 9}\end{array}$ & $\begin{array}{c}\text { At high risk for } \\
\text { AEP } \\
\mathbf{N}=\mathbf{2 9}\end{array}$ & $\begin{array}{c}\text { Depressed } \\
\mathbf{N}=\mathbf{2 2}\end{array}$ \\
\hline Partner/spouse & 30.4 & 31.4 & 33.3 & 27.6 & 22.7 \\
\hline Mom & 13 & 13.7 & 10.3 & 10.3 & 4.5 \\
\hline Dad & 8.7 & 7.8 & 7.7 & 6.9 & 4.5 \\
\hline Sister & 25.1 & 27.5 & 30.8 & 24.1 & 18.2 \\
\hline Brother & 8.7 & 7.8 & 10.3 & 6.9 & 9.1 \\
\hline Myself; I drink alone & 17.4 & 21.6 & 25.6 & 20.7 & 22.7 \\
\hline Grandparent & 2.9 & 2 & 2.6 & 3.4 & 0 \\
\hline Cousin & 24.6 & 27.5 & 25.6 & $37.9 *$ & 31.8 \\
\hline Uncle & 4.3 & 2 & 2.6 & 3.4 & 4.5 \\
\hline Aunt & 7.2 & 2 & 2.6 & 3.4 & 13.6 \\
\hline Friend (girlfriend) & 58 & 66.7 & $71.8 *$ & $79.3 *$ & $40.9 *$ \\
\hline Coworker & 11.6 & 11.8 & 15.4 & 13.8 & 4.5 \\
\hline Other & 7.2 & 7.8 & 10.3 & 6.9 & 0 \\
\hline
\end{tabular}

The most common reasons, in order, that non-drinkers cited for not drinking were children, health, and family. Other reasons included "don't like alcohol", "I'm a recovering alcoholic", and "lost loved ones to alcohol related incidents". Current drinkers, when choosing not to drink, reported doing so because of children, having to drive or having to work or go to school. Risky drinkers cited "no money" significantly more often than non-risky drinkers. Women at high risk for an AEP were significantly more likely to credit having to work or drive. Women identified as depressed were significantly more likely to say they choose not drink because they "don't like alcohol”.

The top reasons cited for why participants binge drink were "to relax", "to help me enjoy time with friends or partner", "because of stress", and "to get buzzed". Subgroups differed slightly with risky drinkers significantly more likely to drink to relax $(p<0.001)$ or escape their problems $(p=0.007)$ than non-risky drinkers. Depressed women were more likely than non-depressed women to drink to escape 
their problems $(p=0.010)$, or because of stress $(p=0.004)$ or trauma $(p=0.013)$. They were more likely to have been exposed to sexual assault $(\mathrm{p}=0.032)$.

Depressed women were slightly less likely $(p=.024)$ than non-depressed women to regret drinking during pregnancy when imagining that they had given birth to a child with FASD, and slightly less likely ( $\mathrm{p}=.008)$ to worry about their baby being harmed if they were to consume alcohol while pregnant (data not shown).

Of the 80 women in the paper-and-pencil subgroup sample, 17 declined to answer the abuse/trauma questions. Among the participants responding, 44.4\% had been exposed to physical abuse, $50.8 \%$ to emotional abuse, and $61.9 \%$ to verbal abuse. Thirty-seven percent (36.5\%) had been exposed to trauma and $36.5 \%$ had witnessed a traumatic event. Of the entire responding sample, $27 \%$ had been exposed to sexual assault. This did not differ by current drinker status, which was true for all abuse/trauma questions, but women identified as depressed were significantly more likely to have experienced sexual assault.

Regression analysis reveals that approximately $40 \%$ of variation in the amount alcohol women consumed per week could be explained by their perception of risk to a potential pregnancy, their perception of the drinking patterns of other women, how much their drinking partner drinks, whether they smoke, and whether they feel pressured to drink or not.

\section{Limitations}

Data in this study were self-reported and may have been biased by social acceptability. In view of this possibility, we strove to ensure and convey confidentiality by a variety of means including a Certificate of Confidentiality from the NIH. Approval and support was obtained from the Tribal IRB. Preparation of materials (detailed elsewhere, Montag, et al., 2017), recruitment, and interactions with participants were carried out by trusted community members trained as research staff. Participants were self-selected volunteers and may not represent all women of child-bearing age within the community. However, recruitment occurred at locations throughout the community to allow equal access for all. Validity may have been threatened by our use of 2-week increments when assessing alcohol consumption. A longer period of time may have provided more precise data.

\section{Discussion}

High risk drinking is increasing among women in the US including AIAN women (Grant et al., 2017). To maximize the benefit of interventions to reduce risky drinking and prevent FASD, it may be prudent to target efforts to address specific risk and protective factors among differing subgroups.

In the present study, the relevance of specific risk and protective factors varied depending upon alcohol consumption and depression status. Some factors found to affect drinking in general population 
samples did not do so in the present sample including age, employment, religiosity, and being married or cohabitating. Other factors identified elsewhere as important were also found to be important in our study such as depression, smoking, and trauma. The strength of our finding that women's drinking is influenced by female friends and family members is interesting. As Table 4 shows, the influence of female friends grows with increased risk of harmful alcohol consumption. We did not specify "cousin" as male or female but, consistent with our other findings, many women verbally reported drinking with female cousins.

How do women make choices regarding alcohol consumption? As in previous literature, healthcare provider advice was not cited as a reason to abstain in the present study and appears to be largely ignored (O'Connor \& Whaley, 2006b). Decision making strategies of drinkers and non-drinkers may differ. Reasons cited by non-drinkers for not drinking tended to reflect a more well thought-out or long-term strategy than those cited by current drinkers.

Among study participants, 115 women (34.2\%) were identified as depressed (36.7\% nondrinkers, $31.4 \%$ current drinkers, NS). This rate is high; comparable studies using the same depression instrument found a national prevalence of $14 \%$ (Farr et al., 2010) and an Alaska Native sample prevalence of $20 \%$ (Dillard, et al., 2012). Depression is an independent risk factor for risky drinking and was found to be meaningful in this study. The good news is that there are effective treatments for moderate and severe depression, and that depressed women may be identified using brief, culturally acceptable, inexpensive screening. Present results are being used to inform community response.

\section{Implications for Prevention}

- Differences in risk and protective factors exist among communities and community subgroups necessitating modification and targeting of interventions to maximize impact

- Two strategies are recommended for the present community based on study results:

1a) Community wide ("girl power") intervention campaign, geared to all women, seeking to shift cultural norms by empowering women to protect friends/female relatives. This would include broad information dissemination regarding the reality of lower community alcohol consumption and risks of drinking to women and pregnancies (FASD), as well as encouraging friend-networks focused on support. Self-assessment of drinking may be encouraged by the campaign and required at each clinic interaction;

2a) Screening for depression to help identify women at increased risk for AEP; and 2b) treating depression to prevent alcohol-exposed pregnancies. 


\section{Conclusion}

Risk and protective factors varied among community subgroups. In our sample, girlfriends and female relatives played an important role in influencing the level of risk associated with alcohol consumption. Their impact could be harmful or protective. Perception of community drinking norms was skewed in that participants at greater risk believed the amount of drinking by their peers to be greater. Protective factors, beyond the perception of a lower social norm of drinking, included knowledge of the risks of alcohol consumption, drinking with people who consume less alcohol, and certain decision-making strategies. Consistent with previous literature, children were the top factor inducing AIAN women to stop or reduce drinking (Bezdek et al., 2004).

\section{References}

Allen, J., Mohatt, G. V., Rasmus, S. M., Hazel, K. L., Thomas, L., \& Lindley, S. (2006). The tools to understand: Community as co-researcher on culture-specific protective factors for Alaska Natives. Journal of Prevention \& Intervention in the Community, 32(1-2), 41-59. doi:10.1300/J005v32n01_04

Bakhireva, L. N., Wilsnack, S. C., Kristjanson, A., Yevtushok, L., Onishenko, S., Wertelecki, W., \& Chambers, C. D. (2011). Paternal drinking, intimate relationship quality, and alcohol consumption in pregnant Ukrainian women. Journal of Studies on Alcohol \& Drugs, 72(4), 536544. doi:10.15288/jsad.2011.72.536

Balachova, T., Bonner, B., Chaffin, M., Bard, D., Isurina, G., Tsvetkova, L., \& Volkova, E. (2011). Women's alcohol consumption and risk for alcohol-exposed pregnancies in Russia. Addiction, 107(1), 109-117. doi:10.1111/j.1360-0443.2011.03569.x

Beals, J., Manson, S. M., Whitesell, N. R., Mitchell, C. M., Novins, D. K., Simpson, S., \& Spicer, P. (2005). Prevalence of major depressive episode in two American Indian reservation populations: unexpected findings with a structured interview. American Journal of Psychiatry, 162(9), 17131722. doi:10.1176/appi.ajp.162.9.1713

Beals, J., Spicer, P., Mitchell, C. M., Novins, D. K., \& Manson, S. M., AI-SUPERPFP Team. (2003). Racial disparities in alcohol use: Comparison of 2 American Indian reservation populations with national data. American Journal of Public Health, 93(10), 1683. doi:10.2105/AJPH.93.10.1683

Bezdek, M., Croy, C., \& Spicer, P., \& AI-SUPERPFP Team. (2004). Documenting Natural Recovery in American-Indian Drinking Behavior: A Coding Scheme. Journal of Studies on Alcohol, 65(4), 428-433. doi:10.15288/jsa.2004.65.428

Branco, E., \& Kaskutas, L. (2001). "If it burns going down...": How focus groups can shape fetal alcohol syndrome (FAS) prevention. Substance Use \& Misuse, 36(3), 333-345. doi:10.1081/JA100102629 
Caldwell JY, D. J., Davis, J.D., Du Bois B, Echo-Hawk H, Erickson JS, Goins RT, Hill, C, Hillabrant W, Johnson SR, Kendall E, Keemer K, Manson SM, Marshall CA, Running Wolf P, Santiago RL, Schacht R, Stone, JB. (2005). Culturally Competent Research with American Indians and Alaska Natives: Findings and Recommendations of the First Symposium of the Work Group on American Indian Research and Program Evaluation Methodology. American Indian and Alaska Native Mental Health Research: The Journal of the National Center, 12(1), 1-21. ERIC \# EJ742928

Cannon, M. J., Guo, J., Denny, C. H., Green, P. P., Miracle, H., Sniezek, J. E., \& Floyd, R. L. (2015). Prevalence and characteristics of women at risk for an alcohol-exposed pregnancy (AEP) in the United States: estimates from the National Survey of Family Growth. Maternal Child Health Journal, 19(4), 776-782. doi:10.1007/s10995-014-1563-3

Chang, G. (2001). Alcohol screening instruments for pregnant women. Alcohol Research and Health, 25(3), 204-209, PUBMED ID 11810959.

Chang, G., Wilkins-Haug, L., Berman, S., Goetz, M. A., Behr, H., \& Hiley, A. (1998). Alcohol use and pregnancy: improving identification. Obstetrics \& Gynecology, 91(6), 892-898. doi:10.1016/S0029-7844(98)00088-X

Chiodo, L. M., Sokol, R. J., Delaney-Black, V., Janisse, J., \& Hannigan, J. H. (2010). Validity of the TACE in pregnancy in predicting child outcome and risk drinking. Alcohol, 44(7-8), 595-603. doi:10.1016/j.alcohol.2009.08.009

Davis, J.D., Keemer, K. (2002). A brief history of and future considerations for research in American Indian and Alaska Native Communities. ERIC Database \# ED473270.

Dillard, D. A., Smith, J. J., Ferucci, E. D., \& Lanier, A. P. (2012). Depression prevalence and associated factors among Alaska Native people: The Alaska education and research toward health (EARTH) study. Journal of Affective Disorders, 136(3), 1088-1097. doi:10.1016/j.jad.2011.10.042

Duran, B., Sanders, M., Skipper, B., Waitzkin, H., Malcoe, L. H., Paine, S., \& Yager, J. (2004). Prevalence and correlates of mental disorders among Native American women in primary care. American Journal of Public Health, 94(1). doi:10.2105/AJPH.94.1.71

Ethen, M. K., Ramadhani, T. A., Scheuerle, A. E., Canfield, M. A., Wyszynski, D. F., Druschel, C. M., \& Romitti, P. A. (2009). Alcohol consumption by women before and during pregnancy. Maternal and Child Health Journal, 13(2), 274-285. doi:10.1007/s10995-008-0328-2

Farr, S. L., Bitsko, R. H., Hayes, D. K., \& Dietz, P. M. (2010). Mental health and access to services among US women of reproductive age. American Journal of Obstetrics and Gynecology, 203(6), 542. e1-542. e9. doi:10.1016/j.ajog.2010.07.007

Fisher, T. L., Burnet, D. L., Huang, E. S., Chin, M. H., \& Cagney, K. A. (2007). Cultural leverage interventions using culture to narrow racial disparities in health care. Medical Care Research and Review, 64(5 suppl), 243S-282S. DOI 10.1177/1077558707305414.

Flynn, H. A., \& Chermack, S. T. (2008). Prenatal alcohol use: the role of lifetime problems with alcohol, drugs, depression, and violence. Journal of Studies on Alcohol and Drugs, 69(4), 500-509. doi:10.15288/jsad.2008.69.500 
Gale, T., White, J., \& Welty, T. (1998). Differences in detection of alcohol use in a prenatal population (on a Northern Plains Indian Reservation) using various methods of ascertainment. South Dakota Journal of Medicine, 51(7), 235.

Gone, J. P., \& Trimble, J. E. (2012). American Indian and Alaska Native mental health: Diverse perspectives on enduring disparities. Annual Review of Clinical Psychology, 8, 131-160. doi:10.1146/annurev-clinpsy-032511-143127

Grant, B. F., Chou, S. P., Saha, T. D., Pickering, R. P., Kerridge, B. T., Ruan, W. J., . . Fan, A. (2017). Prevalence of 12-month alcohol use, high-risk drinking, and DSM-IV alcohol use disorder in the United States, 2001-2002 to 2012-2013: results from the National Epidemiologic Survey on Alcohol and Related Conditions. Jama Psychiatry, 74(9), 911-923. doi:10.1001/jamapsychiatry.2017.2161

Gray, N., Mays, M. Z., Wolf, D., \& Jirsak, J. (2010). A Culturally Focused Wellness Intervention for American Indian Women of a Small Southwest Community: Associations With Alcohol Use, Abstinence Self-Efficacy, Symptoms of Depression, and Self-Esteem. American Journal of Health Promotion, 25(2), 1-10. doi:10.4278ajhp.080923-QUAN-209

Green PP, M.-E. L., Tan CH, Mejia R, Denny CH. . (2016). Vital Signs: Alcohol-Exposed Pregnancies — United States, 2011-2013. MMWR Morb Mortal Wkly Rep, 65(65), 91-97.

Griner, D., \& Smith, T. B. (2006). Culturally adapted mental health intervention: A meta-analytic review. Psychotherapy, 43(4), 531-554. doi:10.1037/0033-3204.43.4.531

Hawkins, J. D., Van Horn, M. L., \& Arthur, M. W. (2004). Community variation in risk and protective factors and substance use outcomes. Prevention Science, 5(4), 213-220. doi:10.1023/B:PREV.0000045355.53137.45

Helzer, J. E., \& Pryzbeck, T. R. (1988). The co-occurrence of alcoholism with other psychiatric disorders in the general population and its impact on treatment. Journal of studies on alcohol and drugs, 49(03), 219. doi:10.15288/jsa.1988.49.219

Jones, K. L., \& Smith, D. W. (1973). Recognition of the fetal alcohol syndrome in early infancy. Lancet, 2. doi:10.1016/s0140-6736(73)91092-1

Kaskutas, L. (2000). Understanding drinking during pregnancy among urban American Indians and African Americans: health messages, risk beliefs, and how we measure consumption. Alcoholism: Clinical and Experimental Research, 24(8), 1241-1250. doi:10.1111/j.15300277.2000.tb02090.x

Kessler, R. C., Crum, R. M., Warner, L. A., Nelson, C. B., Schulenberg, J., \& Anthony, J. C. (1997). LIfetime co-occurrence of dsm-iii-r alcohol abuse and dependence with other psychiatric disorders in the national comorbidity survey. Archives of General Psychiatry, 54(4), 313-321. doi:10.1001/archpsyc.1997.01830160031005

Kreuter, M. W., Oswald, D. L., Bull, F. C., \& Clark, E. M. (2000). Are tailored health education materials always more effective than non-tailored materials? Health Education Research, 15(3), 305-315. 
Kroenke, K., \& Spitzer, R. L. (2002). The PHQ-9: a new depression diagnostic and severity measure. Psychiatric Annals, 32(9), 509-515. doi:10.3928/0048-5713-20020901-06

Kroenke, K., Spitzer, R. L., \& Williams, J. B. (2001). The PHQ-9. Journal of general internal medicine, 16(9), 606-613. doi:10.1046/j.1525-1497.2001.016009606.x

Kunitz, S. (2006). Life Course Observations of Alcohol Use among Navajo Indians: Natural History or Careers? Medical Anthropology Quarterly, 20(3), 279-296. doi:10.1525/maq.2006.20.3.279

KUO, P.-H., GARDNER, C. O., KENDLER, K. S., \& PRESCOTT, C. A. (2006). The temporal relationship of the onsets of alcohol dependence and major depression: using a genetically informative study design. Psychological Medicine, 36(08), 1153-1162. doi:doi:10.1017/S0033291706007860

LaFromboise, T. D., Hoyt, D. R., Oliver, L., \& Whitbeck, L. B. (2006). Family, community, and school influences on resilience among American Indian adolescents in the upper Midwest. Journal of Community Psychology, 34(2), 193-209. doi:10.1002/jcop.20090

May, P. A., Blankenship, J., Marais, A.-S., Gossage, J. P., Kalberg, W. O., Barnard, R., . . Seedat, S. (2013). Approaching the Prevalence of the Full Spectrum of Fetal Alcohol Spectrum Disorders in a South African Population-Based Study. Alcoholism: Clinical and Experimental Research, 37(5), 818-830. doi:10.1111/acer.12033

May, P. A., \& Gossage, J. P. (2001). New data on the epidemiology of adult drinking and substance use among American Indians of the northern states: male and female data on prevalence, patterns, and consequences. American Indian and Alaska Native Mental Health Research, 10(2), 1-26.

May, P. A., Gossage, J. P., Marais, A. S., Adnams, C. M., Hoyme, H. E., Jones, K. L., . . Kalberg, W. O. (2007). The epidemiology of fetal alcohol syndrome and partial FAS in a South African community. Drug and alcohol dependence, 88(2), 259-271. doi:10.1016/j.drugalcdep.2006.11.007

May, P. A., Gossage, J. P., Marais, A. S., Hendricks, L. S., Snell, C. L., Tabachnick, B. G., ... Viljoen, D. L. (2008). Maternal risk factors for fetal alcohol syndrome and partial fetal alcohol syndrome in South Africa: a third study. Alcoholism: Clinical and Experimental Research, 32(5), 738-753.

May, P. A., Gossage, J. P., White-Country, M., Goodhart, K., Decoteau, S., Trujillo, P. M., .. . Hoyme, H. E. (2004). Alcohol consumption and other maternal risk factors for fetal alcohol syndrome among three distinct samples of women before, during, and after pregnancy: The risk is relative. American Journal of Medical Genetics Part C: Seminars in Medical Genetics, 127C(1), 10-20. doi:10.1002/ajmg.c.30011

May, P. A., \& Phillip Gossage, J. (2011). Maternal risk factors for fetal alcohol spectrum disorders: Not as simple as it might seem. Alcohol Research and Health, 34(1), 15. doi:1535-7414

McLeod, J. D. (1993). Spouse concordance for alcohol dependence and heavy drinking: evidence from a community sample. Alcohol Clin Exp Res, 17(6), 1146-1155. doi:10.1111/j.15300277.1993.tb05220.x

Montag, A., Clapp, J. D., Calac, D., Gorman, J., \& Chambers, C. (2012). A review of evidence-based approaches for reduction of alcohol consumption in native women who are pregnant or of 
reproductive age. The American journal of drug and alcohol abuse, 38(5), 436-443.

doi:10.3109/00952990.2012.694521

Montag, A. C., Brodine, S. K., Alcaraz, J. E., Clapp, J. D., Allison, M. A., Calac, D. J., . . Chambers, C. D. (2015). Effect of depression on risky drinking and response to a screening, brief intervention, and referral to treatment intervention. American journal of public health, 105(8), 1572-1576. doi:10.2105/AJPH.2015.302688

Montag, A. C., Dusek, M. L., Ortega, M. L., Camp-Mazzetti, A., Calac, D. J., \& Chambers, C. D. (2017). Tailoring an Alcohol Intervention for American Indian Alaska Native Women of Childbearing Age: Listening to the Community. Alcoholism: Clinical and Experimental Research, 41(11), 1938-1945. doi:10.1111/acer.13485

Naimi, T. S., Lipscomb, L. E., Brewer, R. D., \& Gilbert, B. C. (2003). Binge Drinking in the Preconception Period and the Risk of Unintended Pregnancy: Implications for Women and Their Children. Pediatrics, 111(Supplement 1), 1136-1141.

NIAAA. (2011). Women and Alcohol. Retrieved from http://pubs.niaaa.nih.gov/publications/womensfact/womensfact.htm

Nilsen, P., Holmqvist, M., Hultgren, E., Bendtsen, P., \& Cedergren, M. (2008). Alcohol use before and during pregnancy and factors influencing change among Swedish women. Acta Obstetricia et Gynecologica Scandinavica, 87(7), 768-774. doi:doi:10.1080/00016340802179830

O'Connell, J., Novins, D. K., Beals, J., Croy, C., Barón, A. E., Spicer, P., \& Buchwald, D. (2006). The relationship between patterns of alcohol use and mental and physical health disorders in two American Indian populations. Addiction, 101(1), 69-83. doi:10.1111/j.1360-0443.2005.01308.x

O'Connor, M. J., \& Whaley, S. E. (2006a). Health care provider advice and risk factors associated with alcohol consumption following pregnancy recognition. Journal of studies on alcohol, 67(1), 2231. doi:10.15288/jsa.2006.67.22

O'Connor, M. J., \& Whaley, S. E. (2006b). Health care provider advice and risk factors associated with alcohol consumption following pregnancy recognition. Journal of studies on alcohol and drugs, $67(1), 22$.

O'Keeffe, L. M., Kearney, P. M., McCarthy, F. P., Khashan, A. S., Greene, R. A., North, R. A., . . Kenny, L. C. (2015). Prevalence and predictors of alcohol use during pregnancy: findings from international multicentre cohort studies. BMJ Open, 5(7). doi:10.1136/bmjopen-2014-006323

O’Connell, J. M., Novins, D. K., Beals, J., Spicer, P., \& the, A. I. S. T. (2005). Disparities in Patterns of Alcohol Use Among Reservation-based and Geographically Dispersed American Indian Populations. Alcoholism: Clinical and Experimental Research, 29(1), 107-116. doi:10.1097/01.alc.0000153789.59228.fc

Palma, S., Pardo-Crespo, R., Mariscal, M., Perez-Iglesias, R., Llorca, J., \& Delgado-Rodríguez, M. (2007). Weekday but not weekend alcohol consumption before pregnancy influences alcohol cessation during pregnancy. The European Journal of Public Health, 17(4), 394-399. doi:10.1093/eurpub/ck1259 
Parker, T., Maviglia, M. A., Lewis, P. T., Gossage, J. P., \& May, P. A. (2010). Psychological distress among Plains Indian mothers with children referred to screening for Fetal Alcohol Spectrum Disorders. Substance abuse treatment, prevention, and policy, 5(1), 1-9. doi:10.1186/1747$597 \mathrm{X}-5-22$

Patel, J. S. (2017). Measurement Invariance of the Patient Health Questionnaire-9 (PHQ-9) Depression Screener in US Adults Across Sex, Race/Ethnicity, and Education Level: NHANES. Purdue University.

Riley, E., Infante, M. A., \& Warren, K. (2011). Fetal Alcohol Spectrum Disorders: An Overview. Neuropsychology Review, 21(2), 73-80. doi:10.1007/s11065-011-9166-x

Roozen, S., Peters, G.-J. Y., Kok, G., Townend, D., Nijhuis, J., \& Curfs, L. (2016). Worldwide Prevalence of Fetal Alcohol Spectrum Disorders: A Systematic Literature Review Including Meta-Analysis. Alcoholism: Clinical and Experimental Research, 40(1), 18-32. doi:10.1111/acer.12939

Russell, M., Martier, S. S., Sokol, R. J., Mudar, P., Bottoms, S., Jacobson, S., \& Jacobson, J. (1994). Screening for Pregnancy Risk-Drinking. Alcoholism: Clinical and Experimental Research, 18(5), 1156-1161. doi:10.1111/j.1530-0277.1994.tb00097.x

Russell, M., Martier, S. S., Sokol, R. J., Mudar, P., Jacobson, S., \& Jacobson, J. (1996). Detecting risk drinking during pregnancy: a comparison of four screening questionnaires. American journal of public health, 86(10), 1435-1439. doi:10.2105/AJPH.86.10.1435

Secretary of Health and Human Services. (2000). 10th Special Report to the US Congress on Alcohol and Health National Institute on Alcohol Abuse and Alcoholism.

Skinner, C. S., Campbell, M. K., Rimer, B. K., Curry, S., \& Prochaska, J. O. (1999). How effective is tailored print communication? Annals of behavioral medicine, 21(4), 290. doi:10.1007/BF02895960

Slade, T., McEvoy, P., Chapman, C., Grove, R., \& Teesson, M. (2013). Onset and temporal sequencing of lifetime anxiety, mood and substance use disorders in the general population. Epidemiology and psychiatric sciences, 1-9. doi:10.1017/S2045796013000577

Sokol, R. J., Martier, S. S., \& Ager, J. W. (1989). The T-ACE questions: practical prenatal detection of risk-drinking. American Journal of Obstetrics and Gynecology, 160(4), 863. doi:10.1016/00029378(89)90302-5

Spicer, P., Beals, J., Croy, C. D., Mitchell, C. M., Novins, D. K., Moore, L., . . . Protective Factors Project, T. (2003). The Prevalence of DSM-III-R Alcohol Dependence in Two American Indian Populations. Alcoholism: Clinical and Experimental Research, 27(11), 1785-1797. doi:10.1097/01.alc.0000095864.45755.53

Spitzer, R. L., Kroenke, K., \& Williams, J. B. (1999). Validation and utility of a self-report version of PRIME-MD. JAMA: The Journal of the American Medical Association, 282(18), 1737-1744. doi: $10.1001 /$ jama.282.18.1737 
Streissguth, A. (2007). Offspring effects of prenatal alcohol exposure from birth to 25 years: The Seattle Prospective Longitudinal Study. Journal of Clinical Psychology in Medical Settings, 14(2), 81101. doi:10.1007/s10880-007-9067-6

Szlemko, W., Wood, J., \& Thurman, P. (2006). Native Americans and alcohol: Past, present, and future. The Journal of general psychology, 133(4), 435-451. doi:10.3200/GENP.133.4.435-451

Torres Stone, R. A., Whitbeck, L. B., Chen, X., Johnson, K., \& Olson, D. M. (2006). Traditional practices, traditional spirituality, and alcohol cessation among American Indians. Journal of studies on alcohol, 67(2). doi:10.15288/jsa.2006.67.236

Tsai, J., Floyd, R. L., O'Connor, M. J., \& Velasquez, M. M. (2009). Alcohol use and serious psychological distress among women of childbearing age. Addictive Behaviors, 34(2), 146-153. doi:10.1016/j.addbeh.2008.09.005

Waterson, E., Evans, C., \& MURRAY-LYON, I. M. (1990). Is pregnancy a time of changing drinking and smoking patterns for fathers as well as mothers? An initial investigation. British journal of addiction, 85(3), 389-396. doi:10.1111/j.1360-0443.1990.tb00655.x

Whitbeck, L., Chen, X., Hoyt, D., \& Adams, G. (2004). Discrimination, Historical Loss and Enculturation: Culturally Specific Risk and Resiliency Factors for Alcohol Abuse among American Indians*. Journal of studies on alcohol, 65(4), 409-419. doi:10.15288/jsa.2004.65.409

Yuan, N., Eaves, E., Koss, M., Polacca, M., Bletzer, K., \& Goldman, D. (2010). Alcohol is Something That Been With Us Like a Common Cold: Community Perceptions of American Indian Drinking. Substance Use \&\# 38; Misuse, 45(12), 1909-1929. doi:10.3109/10826081003682115 


\section{Appendix A}

Table 1.

Characterization of Sample by Alcohol Consumption Status

\begin{tabular}{|c|c|c|c|c|c|}
\hline Variable & Not Current Drinker & $\mathrm{n}$ & Current Drinker & $\mathrm{n}$ & $P$ value \\
\hline Age (years) & $28.0 \pm 0.62$ & 161 & $28.9 \pm 0.57$ & 179 & .282 \\
\hline Has had a child & 67.7 & 161 & 64.0 & 178 & .479 \\
\hline Pregnancies (number) & $2.44 \pm 0.20$ & 154 & $1.86 \pm 0.15$ & 176 & .018 \\
\hline Children (number) & $1.63 \pm 0.14$ & 161 & $1.44 \pm 0.11$ & 178 & .269 \\
\hline Wants more children & 59.5 & 148 & 59.8 & 174 & .955 \\
\hline Employed & 35.0 & 160 & 50.3 & 173 & .005 \\
\hline Religious & 84.8 & 151 & 87.3 & 166 & .507 \\
\hline Cohabitating & 43.8 & 160 & 42.4 & 177 & .799 \\
\hline Birth Control Effectiveness & & 145 & & 161 & $<.001$ \\
\hline High & 9.0 & & 17.4 & & \\
\hline Medium High & 22.1 & & 35.4 & & \\
\hline Medium Low & 25.5 & & 20.5 & & \\
\hline Low & 0 & & 1.2 & & \\
\hline No birth control & 43.4 & & 25.4 & & \\
\hline Using Birth Control Correctly & 72.2 & 133 & 83.9 & 155 & .016 \\
\hline Smoker & 26.3 & 160 & 38.2 & 178 & .019 \\
\hline Taking illegal drugs & 8.1 & 161 & 15.1 & 172 & .046 \\
\hline Taking prescription drugs & 41.3 & 160 & 35.2 & 175 & .273 \\
\hline Depression medication & 9.2 & 131 & 6.5 & 123 & .432 \\
\hline Depressed & 36.7 & 158 & 31.4 & 175 & .310 \\
\hline Functionality impaired & 7.5 & 134 & 5.2 & 154 & .428 \\
\hline Heard of FASD/FAS & 75.8 & 153 & 72.1 & 172 & .446 \\
\hline Know someone affected by FASD/FAS & 36.4 & 154 & 35.7 & 154 & .906 \\
\hline \multicolumn{6}{|c|}{ Alcohol consumption variables } \\
\hline \multicolumn{6}{|l|}{ Total Sample } \\
\hline Age at first drink & $15.4 \pm 0.3$ & 143 & $15.0 \pm 0.3$ & 178 & .423 \\
\hline \multicolumn{6}{|l|}{ Perception of Other Women's Drinking } \\
\hline Drinks per week & $7.13 \pm 0.81$ & 139 & $9.03 \pm 0.72$ & 165 & .080 \\
\hline Drinks per occasion & $3.23 \pm 0.31$ & 140 & $4.43 \pm 0.28$ & 168 & .004 \\
\hline \multicolumn{6}{|l|}{ Depressed Subset } \\
\hline Age at first drink & $15.6 \pm 0.6$ & 55 & $13.9 \pm 0.4$ & 55 & .023 \\
\hline \multicolumn{6}{|l|}{ Perception of Other Women's Drinking } \\
\hline Drinks per week & $7.38 \pm 1.26$ & 49 & $13.4 \pm 1.9$ & 47 & .008 \\
\hline Drinks per occasion & $2.87 \pm 0.34$ & 50 & $4.98 \pm 0.53$ & 51 & .001 \\
\hline
\end{tabular}




\section{Appendix B}

Table 2.

Characterization of Sample by Depression Status

\begin{tabular}{|c|c|c|c|c|c|}
\hline Variable & Not Depressed & $\mathrm{n}$ & Depressed & $\mathrm{n}$ & $\mathrm{P}$ value \\
\hline Age (years) & $28.2 \pm 0.5$ & 221 & $29.1 \pm 0.8$ & 115 & .332 \\
\hline Has had a child & 63.2 & 220 & 73.0 & 115 & .070 \\
\hline Pregnancies (number) & $2.08 \pm 0.15$ & 213 & $2.28 \pm 0.21$ & 114 & .436 \\
\hline Children (number) & $1.45 \pm 0.10$ & 220 & $1.74 \pm 0.15$ & 115 & .111 \\
\hline Wants more children & 58.6 & 210 & 62.2 & 111 & .642 \\
\hline Employed & 45.4 & 218 & 38.4 & 112 & .223 \\
\hline Religious & 87.3 & 205 & 84.4 & 109 & .475 \\
\hline Cohabitating & 46.6 & 219 & 36.8 & 114 & .089 \\
\hline Birth Control Effectiveness & & 201 & & 104 & .038 \\
\hline High & 14.9 & & 11.5 & & \\
\hline Medium High & 29.4 & & 28.8 & & \\
\hline Medium Low & 24.9 & & 16.3 & & \\
\hline Low & 0 & & 1.9 & & \\
\hline No birth control & 30.9 & & 41.4 & & \\
\hline Using Birth Control Correctly & 83.2 & 196 & 67.8 & 90 & .003 \\
\hline Smoker & 29.4 & 221 & 37.7 & 114 & .123 \\
\hline Taking illegal drugs & 8.2 & 219 & 18.0 & 111 & .015 \\
\hline Taking prescription drugs & 31.4 & 220 & 53.6 & 112 & $<.001$ \\
\hline Depression medication & 4.8 & 165 & 14.9 & 87 & .006 \\
\hline Functionality impaired & 1.2 & 169 & 13.9 & 115 & $<.001$ \\
\hline Heard of FASD/FAS & 75.7 & 210 & 70.8 & 113 & .337 \\
\hline Know someone affected by FASD/FAS & 36.1 & 202 & 35.6 & 104 & .923 \\
\hline \multicolumn{6}{|c|}{ Alcohol consumption variables } \\
\hline \multicolumn{6}{|l|}{ Total Sample } \\
\hline Drinks per week & $2.88 \pm 0.35$ & 203 & $6.10 \pm 1.18$ & 108 & .001 \\
\hline Drinks per occasion & $2.31 \pm 0.27$ & 206 & $2.29 \pm 0.37$ & 110 & .972 \\
\hline Binge episodes / 2 weeks & $0.95 \pm 0.12$ & 202 & $2.04 \pm 0.45$ & 108 & .004 \\
\hline Age at first drink & $15.5 \pm 0.3$ & 204 & $14.7 \pm 0.4$ & 110 & .069 \\
\hline \multicolumn{6}{|l|}{ Perception of Other Women's Drinking } \\
\hline Drinks per week & $7.04 \pm 0.58$ & 203 & $10.2 \pm 1.1$ & 98 & .006 \\
\hline Drinks per occasion & $3.78 \pm 0.29$ & 203 & $3.89 \pm 0.33$ & 103 & .805 \\
\hline \multicolumn{6}{|l|}{ Current Drinkers } \\
\hline Drinks per week & $5.15 \pm 0.53$ & 114 & $12.4 \pm 2.1$ & 53 & $<.001$ \\
\hline Drinks per occasion & $4.06 \pm 0.40$ & 117 & $4.67 \pm 0.60$ & 54 & .400 \\
\hline Binge episodes / 2 weeks & $1.64 \pm 0.19$ & 117 & $4.15 \pm 0.83$ & 53 & $<.001$ \\
\hline Age at first drink & $15.6 \pm 0.3$ & 119 & $13.9 \pm 0.4$ & 55 & .002 \\
\hline \multicolumn{6}{|l|}{ Perception of Other Women's Drinking } \\
\hline Drinks per week & $7.07 \pm 0.61$ & 115 & $13.4 \pm 1.9$ & 47 & $<.001$ \\
\hline Drinks per occasion & $4.07 \pm 0.33$ & 114 & $4.98 \pm 0.53$ & 51 & .137 \\
\hline
\end{tabular}




\section{Appendix C.}

\section{Knowledge Questions}

\begin{tabular}{|c|}
\hline $\begin{array}{c}\text { Knowledge Questions } \\
\text { (Percent Answering Correctly) }\end{array}$ \\
\hline Questions Relating to Pregnancy \\
\hline When a woman drinks alcohol when she is pregnant, the alcohol enters the baby's bloodstream. (T) \\
\hline Just having a FEW drinks (1-3) during pregnancy is safe for the baby. (F) \\
\hline $\begin{array}{l}\text { Babies of women who drink alcohol during pregnancy are at risk for developing physical, mental and } \\
\text { behavioral problems. (T) }\end{array}$ \\
\hline Drinking alcohol is OK during the last 3 months of pregnancy. $(\mathrm{F})$ \\
\hline $\begin{array}{l}\text { If a woman is already pregnant but does not know it yet and she is drinking alcohol, she can have a } \\
\text { child with an Alcohol Related Birth Defect. (T) }\end{array}$ \\
\hline During pregnancy, it is $\mathrm{OK}$ to drink during the morning. $(\mathrm{F})$ \\
\hline $\begin{array}{l}\text { If you are breastfeeding and you drink alcohol, the alcohol can be passed to the baby through the milk. } \\
\text { (T) }\end{array}$ \\
\hline It is $\mathrm{OK}$ to drink wine during pregnancy. (F) \\
\hline If you are nauseous or feel sick to your stomach during pregnancy, you should drink a beer. (F) \\
\hline Questions Relating to Women's Health \\
\hline Women are at a greater risk for developing alcohol-related problems than men. (T) \\
\hline Most women aged 18-44 who are members of Southwestern Tribes \\
\hline
\end{tabular}

